\section{Windenergie im Netz}

Das neue Ökostromgesetz hat einen Boom in der Installation der Windenergieanlagen eingeleitet. Niemand kann voraussagen, wie viele Anlagen bis 2008 an das Netz angeschlossen werden. Die Zahl der Anmeldungen ist aber bereits beachtlich und wahrscheinlich werden zwischen $800 \mathrm{MW}$ bis $1700 \mathrm{MW}$ erreicht werden. Die Technologie der Windenergieanlagen hat große Fortschritte erzielt. Heute werden bereits Nennleistungen von $5 \mathrm{MW}$ bei Nabenhöhen von $100 \mathrm{~m}$ und Rotordurchmessern bis $120 \mathrm{~m}$ erreicht. Durch die immer größeren Anlagen sind auch im Binnenland von Österreich Volllaststunden bis 2200 möglich.

Die Windenergie kann in den Landesnetzen mit hoher Erzeugung - dies sind das Burgenland und Niederösterreich - bei mäßiger Erzeugung lokal verbraucht werden. Bei starkem Wind und geringem Verbrauch zur Nachtzeit ist dies nicht möglich, und die Windenergie muss über die Landesnetze zum Übertragungsnetz abgeleitet werden.

Hier kommen in Kürze weitere Probleme auf uns zu. Das Übertragungsnetz ist derzeit bereits im Winter überlastet. Die zusätzliche Windbelastung wird es noch weiter in die Überlast treiben. Es ist dringend erfordertich, die Genehmigungsverfahren für den noch ausstehenden Lückenschluss im 380-kV-Netz zu beschleunigen, um nicht in technisch nicht mehr beherrschbare Situationen zu geraten.

Wind hat einen hohen Bedarf an Ausgleichsenergie, der etwa $15 \%$ bis $20 \%$ der erzeugten Energie ausmacht. Die beiliegenden B́eiträge sollen diese Probleme aufzeigen. Sie behandeln die Anschlussbedingungen, die Engpasssituation und die erforderlichen Ausbaumaßnahmen in den Landesnetzen und im Verbundnetz.

Hoffen wir, dass wir durch besonderes Engpassmanagement und durch den raschen Ausbau des 380-kV-Rings die Probleme beherrschen können. Wie ernst die Situation in unserem Netz bereits ist, haben wir Ende August gesehen, als der Ausfall einer Verbundleitung in Ungarn unser Netz bis zur

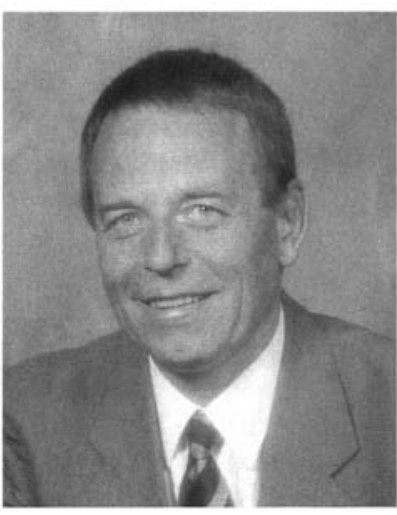

Grenze eines Spannungszusammenbruchs geführt hat, der durch die Auslösung der vorgesehenen Sollbruchstellen gerade noch knapp abgefangen werden konnte.

Windenergie stellt neben Biomasse die hautsächlichen Potenziale an Ökoenergie dar. Beide sind wichtig, um die KyotoZiele zwar nicht zu erfüllen, aber uns ihnen besser anzunähern. Auch aus diesem Grund ist die rasche Netzintegration der Windenergie wichtig.

Anfang nächsten Jahres werden wir in der e \& i einen weiteren Schwerpunkt über Windenergie haben, bei dem wir auch die Technik der Anlagen in ihrer Auswirkung auf das Netz darstellen wollen.

$\mathrm{Ihr}$

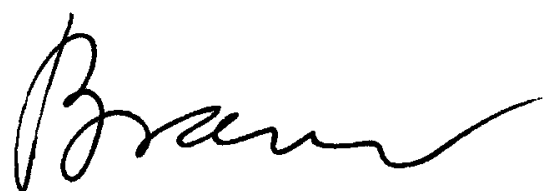

Günther Brauner 